\title{
Corela
}

Cognition, représentation, langage

4-2 | 2006

Vol. $4, n^{\circ} 2$

\section{Les sens procéduraux intersubjectifs de la particule UP}

\section{Fabienne Toupin}

\section{CpenEdition}

\section{Journals}

Édition électronique

URL : http://journals.openedition.org/corela/469

DOI : $10.4000 /$ corela.469

ISSN : $1638-573 \mathrm{X}$

\section{Éditeur}

Cercle linguistique du Centre et de I'Ouest - CerLICO

Référence électronique

Fabienne Toupin, «Les sens procéduraux intersubjectifs de la particule UP », Corela [En ligne], 4-2 | 2006, mis en ligne le 15 décembre 2006, consulté le 20 avril 2019. URL : http:// journals.openedition.org/corela/469; DOI : 10.4000/corela.469

Ce document a été généré automatiquement le 20 avril 2019

\section{(c) (i) (2)(2)}

Corela - cognition, représentation, langage est mis à disposition selon les termes de la licence Creative Commons Attribution - Pas d'Utilisation Commerciale - Partage dans les Mêmes Conditions 4.0 International. 


\title{
Les sens procéduraux intersubjectifs de la particule UP 1
}

\author{
Fabienne Toupin
}

\section{Introduction}

1 Les réflexions essentiellement synchroniques présentées ici constituent le prolongement d'un travail sur la particule UP entamé dans une optique diachronique (cf. Toupin 2006). L'expression même de sens procédural intersubjectif nous est inspirée par la théorie du changement sémantique de Traugott \& Dasher (2002), dite pragmatique historique (nous y référons ci-après par la notation abrégée : Traugott 2002).

2 Il convient de commencer par spécifier le statut théorique des termes de procédural et d' intersubjectif.

3 Selon Traugott et Dasher, les lexèmes suivent universellement une évolution sémantique en trois étapes, qui les conduit de l'expression de la spatialité (expression des relations spatiales) à celle du point de vue du locuteur (ang. subjectification - point de vue étant à prendre au sens large, et non par exemple au sens restreint d'une modalité appréciative) et enfin à l'encodage de l'attitude du locuteur vis-à-vis de son interlocuteur dans le "ici et maintenant" de l'acte de parole (ang. intersubjectification) :

In instances where meanings come explicitly to index and acknowledge SP/W's attitude toward $\mathrm{AD} / \mathrm{R}$ in the here and now of the speech event,

intersubjectification can be said to take place, for example, when non-honorifics are recruited to serve honorific purposes. Intersubjectification, therefore, is a change which results in the development of meanings that explicitly reveal recipient design: the designing of utterances for an intended audience (Clark and Carlson 1982) at the discourse level. (Traugott $2002: 31$ - c'est moi qui souligne) [SP/W $=$ speaker/writer, $\mathrm{AD} / \mathrm{R}=$ addressee/reader $]$

Un sens intersubjectif est donc le produit ultime d'une évolution historiquement programmée ${ }^{2}$ et permet à un lexème de "express grounding in the relationship between speaker/writer and addressee/reader explicitly" (Traugott 2002: 6). Les sens de ce type sont 
d'une nature bien particulière, dans la mesure où il s'agit de sens procéduraux, concept que Traugott et Dasher reprennent aux théoriciens de la pertinence :

Like Relevance Theorists, we also assume that there is a division of labor among Ms [= Meanings]: some are primarily contentful, others primarily procedural (Blakemore 1987). Meanings expressed by nouns, verbs, adjectives, prepositions, and adverbs in some of their uses are usually of the contentful type. By contrast, procedural meanings are primarily indexical of SP/W's attitudes to the discourse and the participants in it; they index metatextual relations between propositions or between propositions and the non-linguistic context. (Traugott 2002: 10 - c'est moi qui souligne)

Ainsi, un sens peut être qualifié de procédural lorsqu'il permet au locuteur d'exprimer un point de vue sur son propre discours et/ou sur la relation qu'il entretient avec son partenaire dans le "ici et maintenant" de l'acte de parole. C'est un sens de dicto, non de re.

De par la définition d'intersubjectification, on voit que l'intersubjectif est nécessairement procédural, mais non l'inverse : un modal, par exemple, pourra avoir un sens procédural subjectif (au sens de subjectification ci-dessus).

7 Nous adoptons ici les hypothèses et concepts de la pragmatique historique, mais avec une conception énonciativiste du sujet parlant et de la relation avec son destinataire dans l'acte de parole. La dyade de référence sera donc pour nous énonciateur/coénonciateur plutôt que locuteur/destinataire - on sait que ce changement n'est pas anodin (Groussier \& Rivière 1996). S'agissant d'intersubjectif, nous reprenons à notre compte l'idée de "index and acknowledge SP/W's attitude toward $A D / R$ in the here and now of the speech event" du premier passage cité, mais en l'investissant du sens qu'elle peut recouvrir dans un cadre énonciativiste, en particulier sous l'impulsion de Claude Delmas (Delmas et al.1993). Quant à procédural, il fait sens dans une optique énonciativiste, en référence au processus de construction de l'énoncé, donc de négociation du sens, entre énonciateur et coénonciateur.

\section{Un premier sens procedural intersubjectif de up}

\section{1.}

8 Dans le cadre de la Théorie métaopérationnelle, Patrick Gettliffe a passé en revue les critères traditionnels pour le placement de la particule dans le cas des verbes à particule (VPa) transitifs ${ }^{3}$. Il a montré l'insuffisance de ces critères et mis en avant un processus énonciatif/discursif qui vaut pour toutes les particules, dont up, et ce quel que soit le sens - spatial ou non - de la particule et de la combinaison (cf. Gettliffe 1990). Nous l'illustrons avec UP :

(1) But he could do one thing. The sonuvabitch could whistle better than anybody I ever heard. He'd be making his bed, or hanging up stuff in the closet - he was always hanging up stuff in the closet - it drove me crazy - and he'd be whistling when he did it [...]. He could take something very jazzy, like "Tin Roof Blues", and whistle it so nice and easy - right while he was hanging stuff up in the closet - that it could kill you. [Gettliffe $1990: 259$ ]

9 En suivant Delmas et al. (1993), nous proposons ici une traduction pragmatique des thèses de l'auteur, dans le respect de sa pensée. Nous dirons ainsi que le placement de la 
particule dans le linéaire de l'énoncé relève d'une véritable stratégie vis-à-vis du coénonciateur :

- la séquence $<\mathrm{V}$-particule-O $>$ permet à l'énonciateur de construire et de présenter une relation $\mathrm{VPa}-$ Objet pour le bénéfice de son coénonciateur ; il notifie ainsi à ce dernier qu'il ne partage pas encore le contenu de sens présenté parce que, lui, énonciateur, est seul responsable de la relation linguistique.Le coénonciateur, sollicité pour coopérer, ne peut donc pas être co-responsable de la structuration. C'est tout cela que recouvre, dans le cadre métaopérationnel, l'expression de PHASE 1 de la relation;

- avec la séquence <V-O-particule>, l'énonciateur indique à son coénonciateur qu'il connaît le contenu de sens présenté, il l'invite à prendre acte du fait qu'il partage déjà l'information (sémantique et grammaticale). Une cause possible de cet état de fait, comme en (1), est que l'énonciateur a présenté la relation à son partenaire dans une antériorité contextuelle (les 2 partenaires ont déjà coopéré).

Dans l'intervalle séparant les 2 occurrences, la simple absence de remise en cause de la relation par le coénonciateur (ainsi, l'acceptation tacite de cette relation dans un dialogue) est ré-interprétée par l'énonciateur comme une acceptation, une co-avalisation de la relation par le coénonciateur. L'énonciateur présente alors son partenaire comme un complice co-responsable de la structuration, c'est un coénonciateur au sens fort du terme (notion de connivence métalinguistique).

11 C'est tout cela que l'on dit lorsqu'on dit que la séquence $<\mathrm{V}-\mathrm{O}$-particule $>$ représente la PHASE 2 de la relation $\mathrm{VPa}-$ Objet. Le vecteur des phases est donc un vecteur des statuts - statut de la relation linguistique, de sa prise en charge par l'énonciateur et, enfin, de l'image de l'interlocuteur. Les trois statuts en question sont solidaires, ils covarient. Voyons à présent un autre exemple :

(2) "You know how the Egyptians buried their dead?" I asked the one kid.

"Naa."

"Well, you should. It's very interesting. They wrapped their faces up in these cloths that were treated with some secret chemical. That way they could be buried in their tombs for thousands of years and their faces wouldn't rot or anything. Nobody knows how to do it except the Egyptians. Even modern science." [Gettliffe 1990 : 223]

L'emploi d'emblée dans le discours de la phase 2 de la relation correspond à une stratégie rhétorique et pragmatique : l'énonciateur utilise un acquis de construction (phase 2 de la relation VPa-Objet), qui ne s'utilise normalement que vis-à-vis d'un coénonciateur qui sait, parce qu'en l'occurrence l'énonciateur estime qu'il devrait savoir (you should [know]). Il "piège" ainsi le coénonciateur en lui transmettant un contenu de sens sur le mode de l'acquis, alors que précisément il ne s'agit pas du rappel d'une relation co-avalisée.

(3) A- "You have to fill in the details here. Have you filled up a form like this

before?"

B- "Oh, you don't need to tell me about either filling forms up, or filling them in -

I fill forms in and up all day long, day in and day out." [Gettliffe $1990: 145]$

Il est vrai que la reprise anaphorique de la relationVPa-Objet n'est pas ici le seul facteur de passage à la phase 2 de la relation : la coordination, d'abord disjonctive avec or, puis conjonctive avec and, impose ce statut dans les deux cas: dans le premier cas, il s'agit d'instaurer un parallélisme syntaxique avec filling them in, où la place de l'objet pronominal est contrainte ; dans le second cas, la contrainte est également syntaxique ${ }^{*} I$ 
fill in and up forms all day long). Mais nous livrons cet exemple pour contrecarrer l'idée, qui circule parfois, que la coordination de particules avec des sens idiomatiques est impossible.

(4) I started toying with the idea, while I kept standing there, of giving old Jane a buzz - I mean calling her long-distance at B.M., where she went, instead of calling up her mother to find out when she was coming home. You weren't supposed to call students up late at night, but I had it all figured out. I was going to tell whoever answered the phone that I was her uncle. [Gettliffe $1990: 239]$ giving old Jane a buzz) qui permet le passage à la phase 2 de la relation.

Les exemples suivants, avec "rétrogradation opérationnelle" (passage d'une phase 2 à une phase 1) rappellent que le vecteur des statuts (statut de la relation, donc de l'image du coénonciateur) est un vecteur de langue, non de discours. Le principe explicatif demeure le même :

(5) "Did you make that decision, John? [...]"

"Oh yes, I decided that."

"And was it the right decision?"

"Yes. I tidied that business up. I've tidied up a number of things. In fact I've tidied

up nearly everything!" [Gettliffe $1990: 263$ ]

(6) "So would I," asserted Freddy, backing his mother up - backing up the spirit of

her remark rather than its substance." [Gettliffe $1990: 275$ ]

En (5), l'acquis de construction que représente "I tidied that business up" est utilisé pour justifier l'assertion précédente : "Yes [it was the right decision]". On pense aux énoncés en bet-ing qui peuvent aussi servir de justification à un acte de langage précédent. L'importance du paramètre co-énonciatif apparaît également dans la suite de cet exemple, où l'énonciateur (le personnage prénommé John) passe à une phase 1 de la relation entre le composé tidy up et son objet. Ce dernier a changé : il ne s'agit plus de that business mais, par degrés de généralisation croissants, de a number of things puis de nearly everything; la forme verbale a elle aussi changé - present perfect et non plus prétérit ; autrement dit, l'énonciateur ne parle plus de la décision particulière sur laquelle l'interrogeait son partenaire au début, il fait une opération de bilan, laquelle porte référentiellement sur sa propre vie. Ce bilan est un éclairage nouveau, non partagé par le coénonciateur, et donc introduit pour son information. C'est ce que traduit en surface ce processus que nous avons appelé plus haut de "rétrogradation opérationnelle". Semblablement, en (6), le "dossier clos" que représente "backing his mother up" est réouvert pour introduire un nouvel éclairage, une précision pour le bénéfice du coénonciateur.

\subsection{Hypothèse sur la genèse du premier sens procédural intersubjectif}

Partons du stade historique dans le développement de up où la particule a acquis le sens de "résultativité". Pour Brinton (1988), la résultativité, c'est-à-dire l'atteinte d'un résultat ou d'un état résultant, est au départ un effet de sens, le fruit d'une combinaison contextuelle entre télicité et perfectivité. C'est donc le produit d'un énoncé à valeur télique dans un contexte de prétérit ou de present perfect. Exemple :

(7) Pat maide dronc up pat win.(c1205 LAY. 14349 [Stratmann])

(The maid drank up the wine) 
Dans Toupin (2006), nous avons tenté de montrer que la complétude construite lexicalement, par exemple par un syntagme nominal sujet ou objet quantifié par ALL, avait sans doute aussi joué un rôle dans la genèse de la résultativité :

(8) ba hit alles up brac. (c1205 LAY. 3077 [Stratmann]

(then it broke everything up.)

21 A partir d'un énoncé comme le (9), nous avons défendu l'idée qu'au plus tôt à la fin du 12 ème et au plus tard au début du $13^{\text {ème }}$ siècle, la résultativité était sans doute devenue un sens à part entière (sens encodé) du lexème UP :

(9) He glent upon Sir Gawen, and gaynly he sayde, 'Now sir, heng up byn ax, pat hat h innogh hewen.' (fin $14^{\text {ème }}$ s., Sir Gawain \& the Green Knight 15/476-77)(He looked at Sir Gawain and said aptly: "Now, sir, hang up your axe, which has hewn enough

[= have done with this business, selon les éditeurs de Sir Gauvain, Tolkien \& Gordon (1925: 88)]

Cet énoncé exprime l'atteinte d'un résultat alors qu'il n'est ni au prétérit ni au present perfect (énoncé impératif) et qu'il ne contient aucun lexème référant, comme ALL, à la complétude d'un procès.

Quoi de plus logique pour l'énonciateur, après avoir constaté l'atteinte d'un résultat ou d'un état résultant, que de diriger l'attention du coénonciateur sur ce résultat ou cet état ? Il nous semble qu'une évolution cruciale de cet ordre a pu se mettre en place dès le moyen-anglais. Pour en présenter les modalités, il est nécessaire de faire un détour préliminaire par l'un des principaux bouleversements dans le passage du vieil-anglais au moyen-anglais : la fixation de l'ordre des mots.

Comme dans le passage du latin au français, ce serait une erreur d'affirmer qu'on est passé d'un système relativement libre (avec SOV prédominant) à un système rigide SVO : selon Olga Fischer, on est en réalité passé d'un système gouverné par des facteurs discursifs (on pourrait dire pragmatiques/énonciatifs) à un système basé sur des facteurs syntaxiques. O. Fischer décrit ainsi les principes organisatoires du vieil-anglais :

[...] ordering principles related to weight (cliticisation) and theme-rheme structure, which are themselves presumably interrelated [...]. Thus, NPs that are light (pronominals) and represent given information (pronominals are usually anaphoric) tend to occur early in the clause, whereas heavy NPs and NPs containing new information tend to occur late. (Fischer 1992 : 373)

et souligne une des premières conséquences de la perte des flexions : au début, l'évolution a renforcé le "multivariable but pragmatic Old English system" (Fischer 1992 : 373) :

With the loss of inflections, the greatest need was for subjects and objects to become distinguishable since both were normally represented by NPs. Because only pronominal subjects and objects could still be distinguished in case, SOV order first disappeared in the case of nominal NPs [...]. This, in a way, reinforced the old English trends noted above, but, as it were, only by accident. (Fischer $1992: 374$ - c'est moi qui souligne)

Revenons au verbe à particule. En moyen-anglais, d'une part, en application directe de ce qui précède, la position pré-verbale de l'objet représenté par un SN se raréfie. D'autre part, et pour d'autres raisons, la position pré-verbale de la particule se perd et la situation se simplifie : $c^{\prime}$ est $<\mathrm{V}+\ldots+$ particule $>$ ou $<\mathrm{V}+$ particule...$+>$ que l'on trouve en tout type de proposition ${ }^{4}$. Et finalement, la situation du verbe à particule en moyen-anglais ressemble assez vite, à peu de choses près, à celle de l'anglais moderne :

- si $\mathrm{O}=$ pronom, alors séquence $<\mathrm{V}-\mathrm{O}-$ particule $>$ privilégiée ; 
- si $\mathrm{O}=\mathrm{SN}$, alors séquence $<\mathrm{V}$-particule- $\mathrm{O}>$ ou $<\mathrm{V}-\mathrm{O}$-particule $>$, le second schéma étant moins fréquent (Rissanen $1992: 269$; Fischer et al. 2002 : 205).

Cette époque charnière qu'est le moyen-anglais, moment où la position pré-verbale de la particule se perd et où simultanément s'opère le renforcement des principes discursifs/ pragmatiques du vieil-anglais est pour nous le site temporel idéal de l'évolution vers les sens de type intersubjectif. Le site contextuel de cette évolution pourrait avoir été constitué par les énoncés contenant un verbe transitif en combinaison avec UP, avec objet [+effectum] (cf. OED s.v. UP $\operatorname{adv}^{1}$, \$4.d), l'état de choses résultant ayant un caractère étonnant, important (voire excessif en certains contextes) aux yeux de l'énonciateur :

(10) Priam ... byld up a bygge towne of pe bare urthe. (c1400 Destr. Troy 1535 [OED])

(Priam ... built up a large town out of the bare earth.)

Il y a intersubjectivité au sens où l'énonciateur attire l'attention du coénonciateur sur le résultat produit. Cela se fait par le placement du SN objet en position finale de phrase, par application des principes discursifs/pragmatiques hérités (préservés) du vieil-anglais.

Notre hypothèse est que ce mécanisme s'est ensuite affranchi, par "a shift from de re to de dicto meaning" (Traugott 2002 : 40), de la problématique de la résultativité, et qu'il a alors été possible pour l'énonciateur de le réinvestir pour diriger l'attention du coénonciateur sur la relation linguistique $\mathrm{VPa}-$-objet elle-même, sur son statut.

Dans le passage du vieil-anglais au moyen-anglais, on sait que finalement les facteurs syntaxiques ont pris le pas sur les facteurs discursifs. Mais force est de constater que le système du verbe à particule est un domaine où se lit encore l'ancienne organisation de la langue anglaise, basée sur la primauté des facteurs discursifs/pragmatiques, et non syntaxiques ${ }^{5}$. Et c'est bien pour cela qu'un objet pronominal, comme tous les linguistes le signalent, peut malgré tout se trouver en position finale de séquence :

(11) When we were only acquaintances, you let me be myself, but now you're always protecting me [...]. You may understand beautiful things, but you don't know how to use them; and you wrap yourself up in art and books and music, and would try to wrap up me. [Gettliffe $1990: 271$ ]

\section{Second sens procedural intersubjectif de up}

31 Contrairement au précédent, ce second sens semble spécifique à UP, et on peut le mettre en lumière à partir de ce qu'écrit Henri Adamczewski sur cette particule.

Adamczewski (2003) distingue un $\mathrm{UP}_{1}$ adverbial, qui est l'antonyme de down, d'un UP ${ }_{2}$ qui représente la vraie particule à ses yeux : $\mathrm{UP}_{1}$ précise le sens initial du verbe auquel il est mis en apposition, tandis que $\mathrm{UP}_{2}$ est un métaopérateur qui signale une opération sur le verbe (2003 : 27). Ce serait un "découpleur qui isolerait le verbe de son paradigme naturel" (2003 : 30). Par exemple, tandis que le choix du verbe open par l'énonciateur se conçoit par opposition paradigmatique aux autres verbes envisageables à sa place dans le cotexte, open up serait un "verbe absolu, sans complémentaire" (2003: 29) ; autrement dit, selon l'auteur, avec open up,les oppositions paradigmatiques ne jouent plus car ce stade est dépassé : le choix du verbe open relève d'une antériorité opérationnelle, qui peut d'ailleurs être explicite ou non.

Adamczewski ne parle pas de la relation énonciateur - coénonciateur, mais le statut du coénonciateur covariant avec celui de la relation linguistique considérée, on peut montrer comment intervient le pragmatique : 
(12) Indeed, every now and then, he would stoop down, pick some object off the ground, and examine it closely . [...] Other than picking up objects from the street, Stillman seemed to do nothing. [Gettliffe $1990: 259$ ] langue (2003: 56, 58-59). Ceci nous semble être une erreur, pour des raisons qui figurent implicitement dans le travail même d'Adamczewski :

- les énoncés présentant les deux verbes en séquence et qui servent d'appui à la démonstration sont bien la preuve que les combinaisons ont statut de verbe de discours (si un lock up peut venir dans la subséquence d'un lock et être compris en référence à cette occurrence, il est évident que cela ne peut pas être le cas d'un give up ou d'un run up [debts]) ; 
- il en va de même des traductions en français des composés :

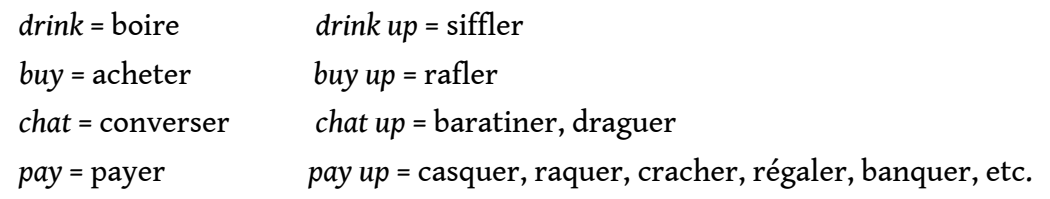
a en anglais contemporain un invariant que l'on peut décrire comme "modulaire" (expression que nous reprenons à Claude Delmas). Cet invariant serait composé de trois grands "modules sémantiques", historiquement engendrés les uns des autres en contexte par un mécanisme d'implicature, et qui correspondent aux trois étapes de l'évolution sémantique dégagées par Traugott et Dasher. Ces modules cohabitent en anglais contemporain, ce qui donne toute sa complexité sémantique à uP - le premier module historique, celui qui permet l'expression des relations spatiales, n'ayant pas disparu avec l'émergence des autres modules ${ }^{7}$. Par ailleurs, les modules ne sont pas plus imperméables les uns aux autres en synchronie qu'ils ne le sont en diachronie : ils forment un continuum qui mène, dans un ordre qui est sans doute un universel, du spatial à l'intersubjectif en passant par l'expression du point de vue de l'énonciateur.

\subsection{Hypothèse sur la genèse du second sens procédural intersubjectif}

Il est possible que le site contextuel de cette évolution ait été constitué par les énoncés impératifs avec verbe divalent, du type de (16) :

(16) "Let me in," I said. "Open the door and let me in."

"I can't do that," the voice answered. "We'll have to talk like this."

I grabbed hold of the door knob and shook the door in frustation. "Open up," I said.

"Open up, or I'll break the door down." [Gettliffe 1990 : 163]

La séquence Open the door! Open up! n'a au départ qu'une valeur d'insistance, pour amener le coénonciateur à réaliser le contenu de l'injonction (insistance sur le résultat souhaité/ projeté). On trouve cette valeur d'insistance dans l'exemple suivant de la fin du $17^{\text {ème }} \mathrm{s}$., ce qui pourrait donner une première indication temporelle sur l'émergence du nouveau sens :

(17) Whatever the verses are, buy 'em upI beseech you. (1682 DRYDEN Medal Ep. Whigs 3[OED])

Corela, 4-2 | 2006 
Tout se passe comme si nous avions Buy them! Buy them up! Dans ce scénario, la valeur d'insistance (sur le résultat à obtenir) aurait été réinterprétée comme une valeur anaphorique (cf. fçs Ouvre! Mais ouvre donc!), ce qui aurait dans un second temps affranchi le mécanisme de la nécessité d'avoir un énoncé impératif :

(18) He lit a cigarette and fell back onto the bed. Perhaps it was because a cigarette cost sixpence and therefore to light it up was an act of calculated extravagance.

[Adamczewski 2003: 42]

et même de la nécessité d'avoir un verbe divalent :

(19) Then he pushed open the door, and the great, pillared gloom was before him, in which his soul shuddered and rose from her nest. His soul leapt, soared up into the great church. His body stood still, absorbed by the height. His soul leapt up into the gloom. [Gettliffe 1990 : 162]

\section{4. bilan}

Comparons les deux sens procéduraux intersubjectifs que nous postulons pour UP. D'un côté, on a :

- un procédé syntaxique (ordre des mots)* qui concerne la relation Verbe à particuleObjet

- un travail commun à toutes les particules (pas uniquement UP) en combinaison avec un verbe transitif, qu'il soit verbe de langue ou de discours, cf. Gettliffe (1990)

- un héritage du vieil-anglais; débuts de ce procédé : fin $12^{\text {ème }} \mathrm{s}$./début13 ${ }^{\mathrm{ème}} \mathrm{s}$. (hypothèse à confirmer)

${ }^{*}$ La séquence $<V$-particule- $O>$ attire l'attention du coénonciateur sur la mise en place de la relation $\mathrm{VPa}-\mathrm{Objet}$, tandis que la séquence $<\mathrm{V}-\mathrm{O}-$ particule $>$ marque un acquis de relation.

De l'autre, on a :

- un procédé morphologique** (simplex vs. composé) qui concerne la relation VerbeCotexte

- un travail comparable à celui de l'article pour le nom et apparemment spécifique à la particule UP en combinaison avec un verbe de discours (qu'il soit transitif ou intransitif), cf. Adamczewski (2003)

- une innovation de l'époque moderne ; débuts de ce procédé : early Modern English (hypothèse ténue, à confirmer)

* Le composé V+UP attire l'attention du coénonciateur sur l'ancienneté de la relation VerbeCotexte, tandis que le verbe simple marque la mise en place de cette relation.

Dans les 2 cas, s'il y a anaphore explicite, up reprend un contenu lexical, pas une référence à une occurrence de procès précédemment construite. Des énoncés comme le (4) (call students up) ou le (12) (pick up objects) l'indiquent sans ambiguïté. Yaguello (1991) parlerait d'anaphorique disréférentiel, Quirk et al. (1985) de substitution.

2 Finalement, lorsque UP a un sens procédural intersubjectif (lequel n'exclut pas, d'ailleurs, un sens de re), tout se passe comme si le sens «into prominence " «to the notice or consideration of a person" (OED s.v. UP ${ }^{2}, \S \S 11$ et 12) venait s'appliquer à une relation linguistique impliquant le verbe à particule : relation VPa-Objet dans un cas, relation Verbe-Cotexte dans l'autre. C'est ce que veut dire procédural intersubjectif : il y a détournement, réinvestissement du sens, du monde de la description vers celui du discours et de la relation énonciateur - coénonciateur. 
53 La pragmatique historique prédit qu'une évolution sémantique de ce genre implique un changement de portée :

Such shifts typically involve increase in scope, from meanings that function at the propositional level (e.g. manner adverbials in event structures), to meaning with scope over the proposition (e.g. sentential adverbials), to meanings with scope over the discourse unit (e.g. adverbials in discourse marker function). (Traugott 2002 : 40)

C'est bien ce que l'on note dans le cas de uP :

- le up spatial, antonyme de Down, modifie le sens du verbe, il porte donc sur un constituant de la proposition;

- le UP marqueur de télicité et, a fortiori, le uP exprimant le point de vue de l'énonciateur, comme par exemple le uP porteur du sens de résultativité, modifie l'interprétation sémantico-référentielle de la relation prédicative ; il porte donc sur la proposition;

- le up procédural intersubjectif signale au coénonciateur, outre son propre statut sur le plan de la connivence métalinguistique, le statut linguistique d'une relation impliquant le verbe à particule ; il porte donc sur le discours (Traugott dirait qu'il est "métatextuel") 9 .

C'est d'ailleurs dans ce seul domaine, celui de la portée sémantique, que l'affirmation de Henri Adamczewski citée plus haut, selon laquelle le "UP abstrait" n'a plus rien à voir avec le uP spatial, se justifie à nos yeux.

On voit se confirmer, au passage, que les verbes à particules sont bien plus qu'un simple moyen d'enrichissement du lexique : l'évolution sémantique de cette particule bien particulière qu'est UP, qui ferait - dans certaines conditions - de certaines combinaisons la contenant des marqueurs pragmatiques de relation entre partenaires énonciatifs, distinguerait ces combinaisons des lexèmes verbaux simples ainsi que des verbes prépositionnels.

\section{BIBLIOGRAPHIE}

\section{Particules et verbes à particule de l'anglais moderne}

ADAMCZEWSKI, Henri, 2003. Du Nouveau sur les phrasal verbs : UP revisited. Précy-sur-Oise : EMA. BOLINGER, Dwight, 1971. The Phrasal Verb in English. Cambridge (Ma) : Harvard University Press. COURTNEY, Rosemary, 1983. Longman Dictionary of Phrasal Verbs. Londres : Longman.

COWIE, A.P. et R. Mackin, 1975. Oxford Dictionary of Current Idiomatic English, vol. 1. Oxford : Oxford University Press.

DEHÉ, Nicole, Ray Jackendoff, Andrew McIntyre et al., 2002. Verb-Particle Explorations. Berlin \& New York: Mouton de Gruyter.

GETTLIFFE, Patrick, 1990. Verbes à particule et structuration de l'énoncé anglais. Thèse de Doctorat non publiée, Université de Paris III. 
LINDNER S.J., 1983. A Lexico-Semantic Analysis of English Verb-Particle Constructions with OUT and UP. Indiana University Linguistics Club.

QUAYLE, Nigel, 1990. La Particule UP en anglais contemporain : approche psychomécanique. Thèse de Doctorat, Université de Paris IV (publiée sous le titre UP et le verbe à particule en anglais contemporain. Lille : Presses universitaires de Lille, 1994).

ROSSI, Nathalie, 1994. L'Expression de la spatialité à travers DOWN. Thèse de Doctorat non publiée, Université de Toulouse-Le Mirail.

\section{Verbes à particule et préfixés en anglais médiéval et/ou dans une perspective diachronique}

BRINTON, Laurel J., 1985. "Iconicity and semantic change : Old English verbal prefixes". Amsterdamer Beiträge zur älteren Germanistik 23 : 55-70.

BRINTON, Laurel J., 1988. The Development of English Aspectual Systems. Cambridge : Cambridge University Press.

DENISON, David, 1985. "The origins of completive up in English". Neuphilologische Mitteilungen 86 : 37-61.

FISCHER, Olga et al., 2000. "Verb-particles in Old and Middle English". The Syntax of Early English. Cambridge : Cambridge University Press : 80-210.

FRASER, Thomas K. H., 1995. "Remarques sur le passage du préverbe au postverbe en vieil-anglais tardif ". A. Rousseau (ed.), Les Préverbes dans les langues d'Europe : introduction à l'étude de la préverbation. Lille : Presses universitaires du Septentrion, 95-104.

GETTLIFFE, Patrick, 1999. Verbes prépositionnels et verbes à particule. Ellipses.

HILTUNEN, R.A., 1983. "Syntactic variation in the early history of the English phrasal verb". S. Jacobson (ed.), Papers from the Second Scandinavian Symposium on Syntactic Variation. Stockholm : Almqvist \& Wiksell : 95-108.

HILTUNEN, R.A., 1984. "On the semantic and lexical development of the 'Phrasal Verb' in Old and Early Middle English". Linguistic and Stylistic Studies in Medieval English : 47-61 (Publications de l'AMAES, 10).

MITCHELL, Bruce, 1978. "Prepositions, adverbs, prepositional adverbs, postpositions, separable prefixes, or inseparable prefixes, in Old English?". Neuphilologische Mitteilungen 79 : 240-57.

TOUPIN, Fabienne, 2006. "Esquisse de l'évolution sémantique de UP à travers ses contextes d'apparition", à paraître dans les Travaux du CIEREC, Publications de l'Université de Saint-Etienne. VISSER, F. Th., 2002 [1963]. An Historical Syntax of the English Language. Part I : Syntactical Units with One Verb. Brill, Leiden, Boston, Cologne : Brill : 597-606.

Autres

ADAMCZEWSKI, Henri et Claude Delmas, 1982. Grammaire linguistique de l'anglais. Armand Colin. DELMAS, Claude et al., 1993. Méthode et pratique de l'explication grammaticale en anglais. Dunod. FISCHER, Olga, 1992. "Syntax". Norman Blake (ed.), The Cambridge History of the English language. Vol. II (1066-1476) : 207-408.

GRICE, Paul H., 1975. "Logic and conversation". Peter Cole et Jerry L. Morgan (eds)., Syntax and Semantics. Vol. 3 : Speech Acts. New York : Academic Press : 43-58. 
GROUSSIER, Marie-Line et Claude Rivière, 1996. Les Mots de la linguistique : lexique de linguistique énonciative. Paris \& Gap : Ophrys.

HOGG, R.M. (general ed.), 1992. The Cambridge History of the English language. Vols. II (1066-1476) et III (1476-1776).

MOSSE, Fernand, 1945. Manuel de l'anglais du Moyen Age I. Aubier-Montaigne.

NEVALEINEN Terttu, 1992. "Early Modern English Lexis and Semantics". Roger Lass (ed.), The Cambridge History of the English language. Vol. III (1476-1776) : 332-458.

PAILLARD, Michel, 2000. Lexicologie contrastive anglais-français. Paris \& Gap : Ophrys.

QUIRK, Randolph et al., 1985. A Comprehensive Grammar of the English Language. Londres : Longman.

RISSANEN Matti, 1992. "Syntax". Roger Lass (ed.), The Cambridge History of the English language. Vol. III (1476-1776) : 187-331.

SWEETSER, Eve, 1990. From Etymology to Pragmatics. Cambridge : Cambridge University Press.

TOLKIEN J.R.R. et E.V. Gordon (eds.), 1952 [1925]. Sir Gawain and the Green Knight. Oxford : Clarendon Press.

TRAUGOTT Elisabeth \& Richard B. DASHER, 2002. Regularity in Semantic Change. Cambridge : Cambridge University Press.

- Dictionnaires :

BOSWORTH, Joseph and T. Northcote Toller ([1898] 1954). An Anglo-Saxon Dictionary. Glasgow, New York, Toronto : Oxford University Press.

Oxford University Press (ed.), 1994. The Oxford English Dictionary, second edition, on compact disk. Oxford, New York : Oxford University Press.

STRATMANN, Francis H., 1958 [1891]. A Middle-English Dictionary. London : Oxford University Press.

\section{NOTES}

1. Je remercie sincèrement les deux relecteurs anonymes de cet article pour leurs remarques et leurs précieux commentaires, qui vont me permettre de prolonger ma réflexion sur une base enrichie. Je suis seule responsable de toute erreur ou omission demeurant dans le présent texte.

2. Notons bien que la théorie ne postule pas que l'étape 2 existe nécessairement après 1 , ni 3 après 2. En revanche, ce qu'elle prédit, c'est que s'il existe effectivement une étape 2, voire une étape 3, elles verront le développement de sens de type subjectif et intersubjectif respectivement. Tel est le sens du concept de prévisibilité/directionnalité du changement sémantique.

3. On trouve une récente synthèse de ces critères dans Dehé (2002:270).

4. Chiffres de Hiltunen (1983) :

Propositions principales en vieil-anglais $:<\mathrm{V}+\ldots+$ particule $>+\langle\mathrm{V}+$ particule $+\ldots\rangle=59 \%$ de son corpus

Propositions principales en moyen-anglais : $\langle\mathrm{V}+\ldots+$ particule $>+<\mathrm{V}+$ particule $+\ldots>=96 \%$ de son corpus.

5. Nous ne voulons en aucun cas signifier que l'ancienne organisation de la langue anglaise était asyntaxique, tandis que la nouvelle serait "a-discursive".

6. Voir les pages sur l'article anglais, rédigées par Claude Delmas dans Adamczewski et Delmas (1982: 205-21). 
7. Avec up le spatial est rémanent, mais avec be+-ing par exemple, le spatio-temporel est totalement perdu, be+-ing n'a plus à nos yeux que les deuxième et troisième modules.

8. Dans cet exemple, comme dans le (14) plus haut, on note que le passage du verbe simple au composé avec UP correspond à une transformation en verbe inergatif. Nous sommes redevable de cette remarque à J.-C. Khalifa, même si nous ne savons pas pour l'instant comment exploiter cette piste syntaxique.

9. Ces remarques sur la portée de updemandent à être affinées en intégrant la distinction supplémentaire verbe de langue / verbe de discours, dont les conséquences en termes de portée ont déjà été étudiées (Quayle 1990 : 147-151) : composant un verbe de discours, UP est un adverbe et, dans la métalangue de la psychomécanique, il est sémantiquement incident au mouvement qui relie matière verbale et personne d'effection. Elément d'un verbe de langue, uP est un préadverbe dépourvu de capacité incidentielle.

\section{RÉSUMÉS}

Cet article est basé sur les principes de la pragmatique historique ainsi que sur des travaux antérieurs portant sur la particule UP de l'anglais. Il esquisse des hypothèses sur l'existence et la genèse de deux sens procéduraux intersubjectifs que possèderait UP en anglais contemporain. Un de ces sens serait en fait commun à toutes les particules entrant dans la formation de composés transitifs, que le composé soit verbe de langue ou de discours, et ce sens recevrait un marquage syntaxique, grâce à l'ordre des mots retenu pour la triade verbe-particule-objet. L'autre sens, de développement plus récent, serait spécifique à la particule UP entrant dans la formation d'un verbe de discours, qu'il soit transitif ou intransitif, et ce sens serait morphologiquement marqué, grâce au choix d'un verbe simple ou au contraire composé avec UP. Les relations linguistiques impliquées dans chacun des cas ne sont pas les mêmes : relation Verbe à particule-Objet pour le premier sens, Verbe-Cotexte pour le second, mais le point commun est que le statut du coénonciateur covarie avec celui de la relation.

In this paper I adopt the tenets of historical pragmatics and make use of previous scholarly work on the adverbial particle UP to present hypotheses on the existence in PDE and historical development of two intersubjective procedural meanings of the particle.

One of these meanings is not in fact specific to up, but can be expressed by all particles in transitive phrasal verbs. It is encoded syntactically by the word-order adopted by the speaker for organizing verb, particle and object into a sequence.

The other meaning I posit to have developed more recently and to be specific to UP as a component part of a discourse verb, whether transitive or not. It is encoded morphologically by the form chosen by the speaker - compound with UP vs. simplex.

The linguistic relationship at stake in each case is not the same (it is the relation between phrasal verb and object for the first meaning, as opposed to the relation between verb and linguistic context for the second), but in both cases a variation in the form of the relation registers a variation in the speaker's attitude to the addressee. 
INDEX

Mots-clés : particule UP, sens procédural, développement de sens intersubjectifs, pragmatique historique, évolution sémantique, ordre des mots, cotexte, portée

Keywords : particle UP, procedural meaning, intersubjectification, historical pragmatics, word order, semantic development, linguistic context, scope

\section{AUTEUR}

\section{FABIENNE TOUPIN}

Groupe de recherche "Langues \& Représentation", Université François-Rabelais de Tours 